\title{
Ectopic Intrathyroidal Parathyroid Adenoma Presented with Multiple Lytic Bone Lesions
}

\author{
Fatin Hayyani Mohamad Najib ${ }^{1}$, Siti Zarina Amir Hassan², Mohd Ali Emran ${ }^{3}$, Wan Fatihah Wan Sohaimi ${ }^{4}$, Norazlina Mat Nawi ${ }^{5}$
}

\section{Abstract}

We report a rare case of intrathyroidal parathyroid adenoma (ITPA) of a 17-year-old girl with ectopic ITPA with diagnostic and therapeutic challenges due to the rare ectopic location and association with multiple lytic bone lesions. Parathyroid adenoma (PA) is the most common cause of primary hyperparathyroidism. It is a benign tumor and is generally presented as solitary adenoma. Identification of ITPA by imaging characteristics is sometimes difficult, being often an intraoperative discovery that requires special surgical strategy.

Keywords: Brown tumor, Ectopic intrathyroidal parathyroid adenoma, Parathyroid adenoma, Primary hyperparathyroidism. World Journal of Endocrine Surgery (2020): 10.5005/jp-journals-10002-1294
\end{abstract}

\section{BACKGROUND}

Hyperparathyroidism is a disease characterized by excessive secretion of parathyroid hormone (PTH). Hyperparathyroidism is usually subdivided into primary, secondary, and tertiary hyperparathyroidism.

Parathyroid adenoma (PA) is the most common cause of primary hyperparathyroidism, with other rare etiologies being parathyroid hyperplasia and parathyroid carcinoma. Parathyroid adenoma is a benign tumor, generally presented as solitary adenoma of the normally located parathyroid glands. Rarely, ectopic locations, such as, intrathyroidal can be identified. Identification of intrathyroidal parathyroid adenoma (ITPA) by ultrasonography and conventional imaging modalities are sometimes difficult.

We report a case of a 17-year-old girl with multiple lytic bone lesions that leads to a final diagnosis of ectopic solitary ITPA. This case shows diagnostic and therapeutic challenges due to uncommon clinical presentation and final diagnosis of rare ectopic locations.

\section{Case Description}

A 17-year-old girl initially presented with left lower leg swelling and pain for 6 months' duration. She was unable to walk due to pain, requiring crutches to ambulate. There was no prior history of trauma.

On physical examination, there was a mild swelling with an ill-defined margin at the distal half of the left shin, proximal to the left ankle joint associated with tenderness on palpation. No other significant clinical abnormality was observed. Pelvic bone and left tibia/fibula radiographs showed an ill-defined lytic destructive bony lesion with irregular margins seen at the arcuate lining of the right ilium. A similar lesion was also seen at the left superior pubic ramus (Fig. 1).

Laboratory analysis showed significant hypercalcemia as shown in Table 1.

Bone biopsy of the lesion at distal left tibia was done and pathological analysis confirmed the diagnosis of brown tumor. Because of laboratory analysis resulting in hyperparathyroidism supported by histopathological evidence of brown tumor, further evaluation was done to investigate the cause of hyperparathyroidism.
${ }^{1-3}$ Department of Nuclear Medicine, Kuala Lumpur Hospital, Kuala Lumpur, Malaysia

4,5 Department of Nuclear Medicine, Radiotherapy and Oncology, School of Medical Sciences, Universiti Sains Malaysia; Department of Nuclear Medicine, Radiotherapy and Oncology, Hospital Universiti Sains Malaysia (HUSM), Kubang Kerian, Kelantan, Malaysia

Corresponding Author: Wan Fatihah Wan Sohaimi, Department of Nuclear Medicine, Radiotherapy and Oncology, School of Medical Sciences, Universiti Sains Malaysia; Department of Nuclear Medicine, Radiotherapy and Oncology, Hospital Universiti Sains Malaysia (HUSM), Kubang Kerian, Kelantan, Malaysia, Phone: +6097676684, e-mail: fatihahkk@usm.my

How to cite this article: Najib FHM, Hassan SZA, Emran MA, et al. Ectopic Intrathyroidal Parathyroid Adenoma Presented with Multiple Lytic Bone Lesions. World J Endoc Surg 2020;12(2):86-89.

Source of support: USM short-term research grant: (304/PPSP/ 61313140).

Conflict of interest: None

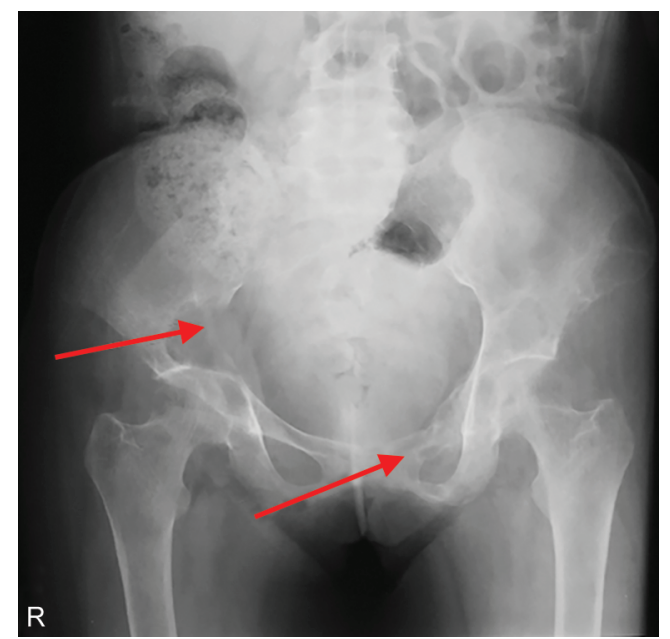

Fig. 1: Pelvic radiograph showed ill-defined lytic destructive bony lesion with irregular margins seen at arcuate lining of right ilium. Similar lesion was also seen at left superior pubic ramus 
Table 1: Laboratory analysis

\begin{tabular}{llll}
\hline Test & Result & Normal value & Interpretation \\
\hline Serum calcium & $3.06 \mathrm{mmol} / \mathrm{L}$ & $2.0-2.55 \mathrm{mmol} / \mathrm{L}$ & High (hypercalcemia) \\
Serum albumin & $48 \mathrm{gm} / \mathrm{L}$ & $32-45 \mathrm{gm} / \mathrm{L}$ & Slightly increased \\
Urine calcium level & $3.7 \mathrm{mmol} / \mathrm{L}$ & $1.7-5.0 \mathrm{mmol} / \mathrm{L}$ & Normal \\
24-hour calcium excretion & $6.7 \mathrm{mmol} / 24$ hours & $15-20 \mathrm{mmol} / 24$ hours & Low \\
Serum phosphate & $0.44 \mathrm{mmol} / \mathrm{L}$ & $1.3-1.9 \mathrm{mmol} / \mathrm{L}$ & Low \\
Serum alkaline phosphatase & $1478 \mathrm{IU} / \mathrm{L}$ & $41-119 \mathrm{IU} / \mathrm{L}$ & High \\
Serum intact parathyroid hormone (iPTH) & $52 \mathrm{pmol} / \mathrm{L}$ & $1.2-5.8 \mathrm{pmol} / \mathrm{L}$ & High \\
\hline
\end{tabular}

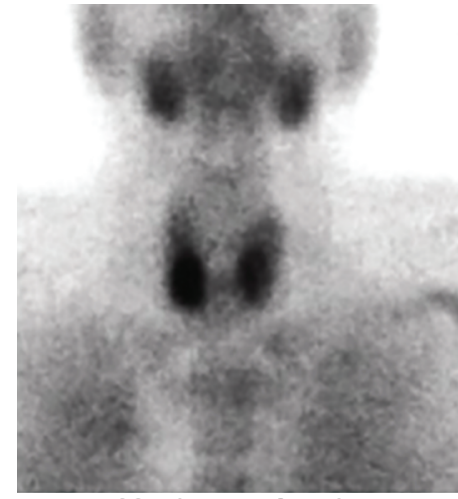

30 minutes of study

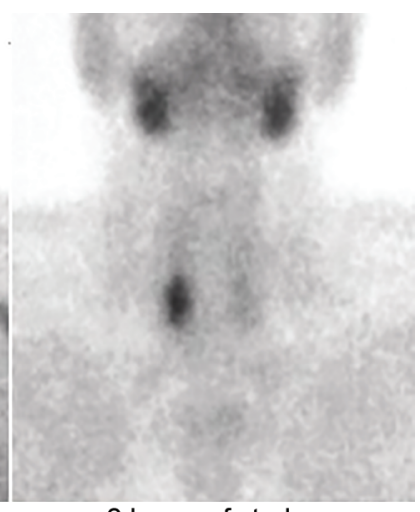

2 hours of study
Fig. 2: Parathyroid scintigraphy with Tc-99m sestamibi. Anterior static views of neck and chest were taken at 30 minutes and 2 hours post-injection. In the early phase (30 minutes), there was good and homogenous tracer uptake and distribution in the thyroid and parathyroid glands. In the delay phase ( 2 hours), there was persistent focus of increased tracer uptake in the right thyroid lobe

Ultrasound of the neck showed a right thyroid nodule measuring $0.9 \times 0.9 \times 2.5 \mathrm{~cm}$. Otherwise, no evidence of parathyroid glands enlargement.

Later, the patient underwent parathyroid scintigraphy to localize the hyperfunctioning parathyroid gland. Parathyroid scintigraphy was performed using the dual-phase method with Tc-99m sestamibi. A static view of the neck and chest was taken at early (30 minutes) and delay ( 2 hours) phases. Subsequently, the whole-body planar view with single-photon emission computed tomography/computed tomography (SPECT/CT) of the neck and leg was taken. Parathyroid scintigraphy showed good and homogeneous tracer uptake and distribution seen in the thyroid and parathyroid glands in the early phase. There was a persistent focus of increased tracer uptake in the right thyroid lobe corresponding to intrathyroidal nodule measuring $1.5 \times 1.0$ $\times 2.0 \mathrm{~cm}(\mathrm{AP} \times \mathrm{W} \times \mathrm{CC})$ on $\mathrm{CT}$ (Figs 2 and 3 ). Whole body planar and SPECT/CT of neck/thorax and lower limb showed multiple foci of increased tracer uptake at the right scapula (Fig. 4), proximal of the right tibia, and distal of the left tibia corresponding to multiple expansile lytic bone lesions on CT. Scan findings showed evidence of functioning ITPA in the right thyroid lobe with evidence of brown tumor in the scapula and both tibia.

The patient underwent right hemithyroidectomy in which the right thyroid lobe and the right inferior parathyroid gland were removed. The right superior parathyroid gland and the left superior and inferior parathyroid glands were preserved. The histopathological examination has confirmed the diagnosis of
ITPA at the right inferior parathyroid gland with otherwise benign thyroid tissue.

Postoperatively, the patient shows favorable evolution as the serum levels of calcium and PTH had declined to a level within their respective normal limits. Currently, after almost 1 year post-surgery, there is a marked improvement in the patient's clinical condition and she remains symptom-free.

\section{Discussion}

Since the advent of laboratory screening and imaging modalities, hyperparathyroidism is detected with increasing frequency. Our patient had presented with multiple ill-defined osteolytic lesions which noted on simple radiographs. Considering the patient's age group, the differential diagnosis was made with benign bone tumors, such as, aneurysmal bone cyst, brown tumor, and giant cell tumor; malignant primary bone tumors, such as, Ewing's sarcoma; metastatic bone disease; and other diseases with osteolytic lesions, such as, multiple myeloma.

The osteolytic lesions have turned out to be a brown tumor by a histopathological diagnosis of the bone biopsy. Brown tumor also is known as osteitis fibrosa cystica or rarely as osteoclastoma, is a unior multi-focal benign bone lesion that related to both primary and secondary hyperparathyroidism. Although it is generally seen more frequently in the cases of serious secondary hyperparathyroidism, it is fairly characteristic for primary hyperparathyroidism. The term "Brown tumor" is a misfit because the lesion does not have malignant potential (although is sometimes invasive) and showing itself as a cellular reparative process. This specific characteristic must be differentiated from giant cell primary bone tumors and aneurysmal bone cysts.

According to literature reviews, multiple brown tumor cases associated with primary hyperparathyroidism were first reported by Joyce et al. ${ }^{1}$ The reported prevalence of the brown tumor is $0.1 \%$ and has been reported to occur in $4.5 \%$ of patients with primary hyperparathyroidism. ${ }^{2}$ Such rare and presentation of multiple bone lesions may simulate bone malignancy (bone metastases) as a study has reported that about $90 \%$ of the patients with skeletal metastases present with multiple bone lesions. The most common sites are the hip bones, long bones, clavicle, ribs, and jaw. It is more common over 50 years of age and is three times more frequent in women than in men. As of our case, the patient falls into an uncommon age group (17 years) and the sites of brown tumor were noted at the right scapula, proximal right tibia, and distal left tibia.

Due to an increased level of PTH, the brown tumor shows an enhancement of bone catabolism. It represents the terminal stage of the hyperparathyroidism-dependent bone pathology. Because of the similar radiological features (cyst-like radiolucent) showed by other lesions, the diagnosis can be difficult. Brown tumor appears as 

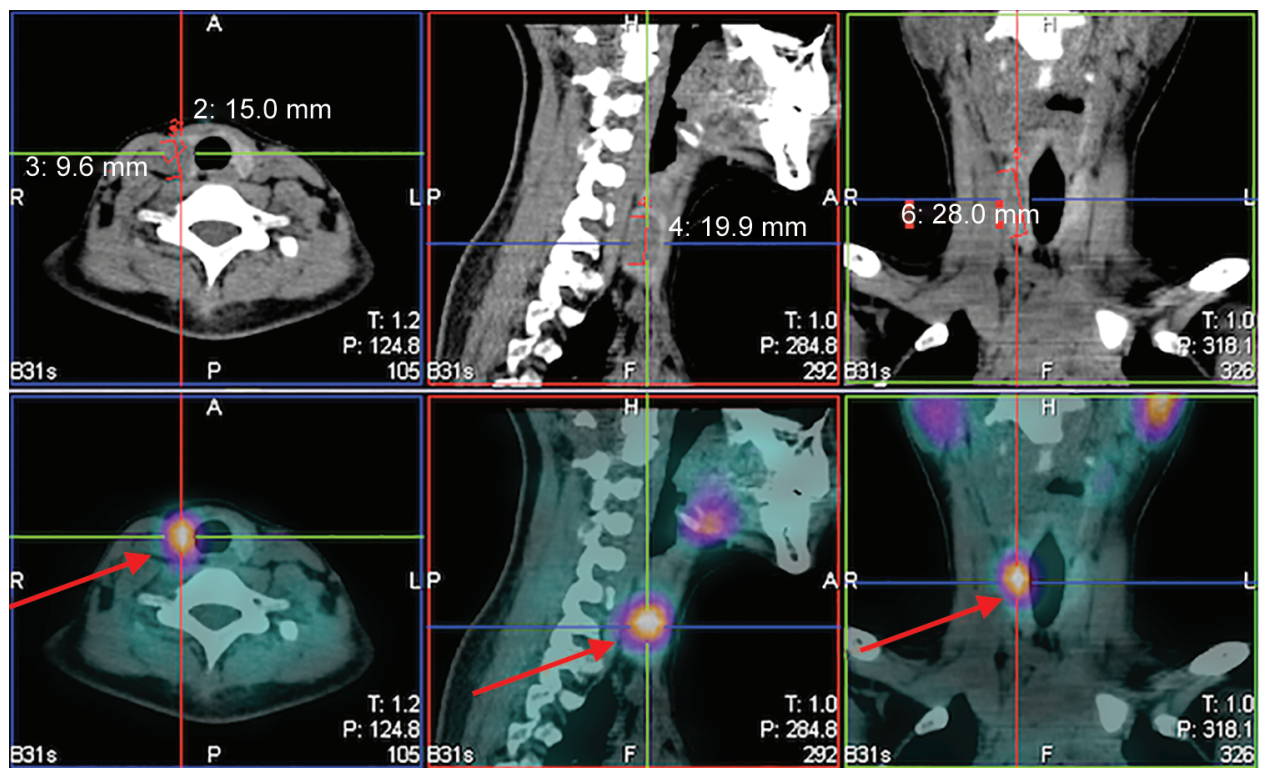

Fig. 3: SPECT/CT of neck/thorax in axial, sagittal and coronal views. The fused images showed focus of increased tracer uptake in the right thyroid lobe corresponding to intrathyroidal nodule measuring $1.5 \times 1.0 \times 2.0 \mathrm{~cm}(\mathrm{AP} \times \mathrm{W} \times \mathrm{CC})$ on $\mathrm{CT}$

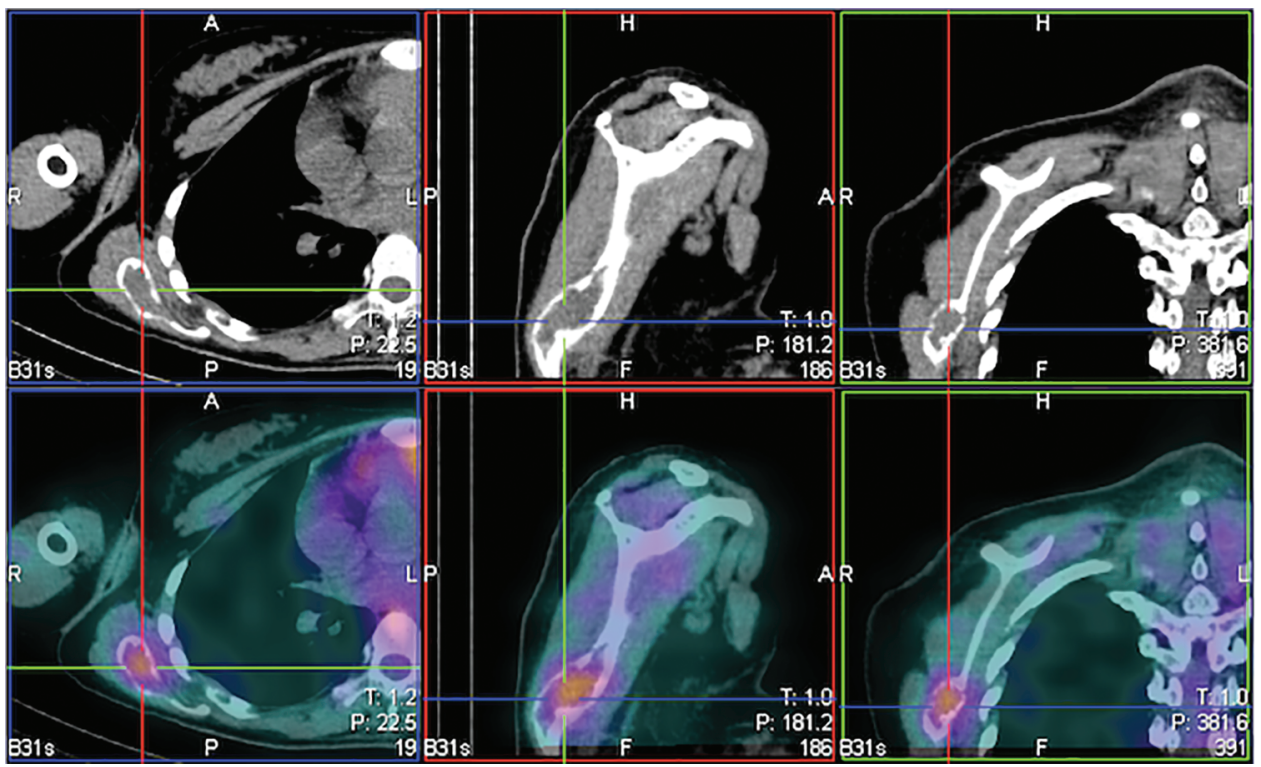

Fig. 4: SPECT/CT of neck/thorax in axial, sagittal and coronal views. The fused images showed focal increased tracer uptake at the inferior angle of right scapula corresponding to expansile lytic bone lesion on CT

a mass with partly cystic and partly solid areas. Microscopically, they are characterized by intensely vascular fibroblastic stroma serving as a background for numerous osteoclast-like multinucleated giant cells. Cysts develop as a result of intraosseous bleeding and tissue degeneration. ${ }^{2}$ Brown tumors may be multiple in the terminal stage of hyperparathyroidism or parathyroid carcinomas. Clinically, they may cause swelling, pathological fracture, and bone pain. ${ }^{3}$

In this case report, further investigations were done toward the causes of hyperparathyroidism. Primary hyperparathyroidism is caused by a PA, hyperplasia, or carcinoma and occurs during the third to fifth decade of life, whereas secondary hyperparathyroidism is often the result of chronic renal failure. Parathyroid adenoma is the most common cause of primary hyperparathyroidism. It is usually presented as a single adenoma of a normally located parathyroid gland.
The incidence of ectopic locations in PA is rare. A study by Phitayakorn and Mchenry has concluded that of 231 patients operated on for hyperparathyroidism, $16 \%$ had ectopic parathyroid glands which were more commonly occur in the inferior parathyroid gland $(62 \%)$ rather than superior parathyroid gland $(38 \%) .{ }^{4}$ Embryologically, the inferior parathyroid tissues are derived from the third branchial pouch and migrate caudally along with the thymus and normally sit near to the inferior pole of the thyroid or adjacent to the thymus; whereas the superior parathyroid tissues are derived from the fourth branchial pouch and normally sit at the posterior to the upper- or midpole of the thyroid gland. Therefore, the ultimate development of ectopic parathyroid tissues is associated with structures related to the third or fourth branchial pouches. Ectopic inferior glands are usually located at intrathymic, anterosuperior mediastinal, intrathyroidal, within the thyrothymic 
ligament and submandibular region, whereas the ectopic superior glands were located in the tracheoesophageal groove, retroesophageal, posterosuperior mediastinal, intrathyroidal, in the carotid sheath and paraesophageal region.

Intrathyroidal parathyroid adenoma is even rarer; their incidence is variable depending on the series of evaluated patients. In a retrospective study on 10,000 patients subjected to surgery for primary hyperparathyroidism, PA was identified in an intrathyroidal position in $0.7 \%$ of cases. A total of 1,163 re-interventions for persistent primary hyperparathyroidism were examined and the adenoma was subsequently found on the lobectomy side in $64 \%$ and on the opposite side in $36 \% .{ }^{5}$ Bahar et al. found that $1.4 \%$ of 426 patients who underwent parathyroidectomy for hyperparathyroidism to have intrathyroidal parathyroid glands. ${ }^{6}$

In this case report, the imaging investigation has begun with an ultrasound examination of the thyroid. The single thyroid nodular mass has raised suspicion of thyroid carcinoma. Ultrasonography is one of the most common imaging methods used for neck evaluation and it is practically the first option in the primary hyperparathyroidism assessment. On ultrasonography, PA is seen typically as round, oval, or polycyclic homogeneous, with hypoechoic structure compared with thyroid parenchyma, surrounded by a thin hyperechoic border (as a capsule) and located outside the thyroid capsule. Morphological differences, such as, hyperechoic component, cystic changes, and calcification, may be seen particularly in large adenomas. More than $90 \%$ of PAs include intraparenchymal hypervascular pattern in the color flow imaging. However, the differential diagnosis is more difficult with a thyroid nodule, especially when goiter coexists. $^{7}$

Ultrasonography evaluation should be completed by scintigraphy, especially in cases where ectopic parathyroid is a suspicion. Parathyroid scintigraphy with Tc-99m sestamibi is a sensitive method for PA identification. It also has a high positive predictive value $(81 \%)$ in the detection of ectopic PA. ${ }^{8}$ Combined parathyroid scintigraphy with SPECT/CT scanners permits more reliable localization of ectopic adenomas. ${ }^{9}$ Although the intrathyroidal location of a PA is rare, in hyperparathyroidism patients with ultrasonography features of normal parathyroid glands and the absence of ectopic parathyroid tissues, SPECT/ CT parathyroid scintigraphy should be considered to detect the possibility of ectopic ITPA.

The surgical option for ITPA is thyroid enucleation or hemithyroidectomy. The preference to perform hemithyroidectomy is to identify and successfully treat an ITPA. In this case report, the patient underwent right hemithyroidectomy resulted in the removal of the right inferior ITPA while preserving the right superior parathyroid gland. The clinical outcome is excellent as the patient remains symptom-free after almost 1 year post-surgery.

\section{Conclusion}

The prevalence of multiple brown tumors is rare and has been related to primary hyperparathyroidism. Nevertheless, in the case of hypercalcemia and radiographic evidence of multiple lytic lesions, primary hyperparathyroidism should always be kept in differential diagnosis and should be looked into once more common causes, such as, malignancy has been excluded. A high index of suspicion will lead to an early diagnosis of the cause of hyperparathyroidism therefore leads to early and prompt treatment.

The preoperative localization of ectopic PA using ultrasonography and parathyroid scintigraphy with SPECT/CT can help to guide the surgeon for the best surgical approach with a favorable clinical outcome, especially in the case of ITPA.

\section{References}

1. Joyce JM, Idea RJ, Grossman SJ, et al. Multiple brown tumours in unsuspected primary hyperparathyroidism mimicking metastatic disease on radiograph and bone scan. Clin Nucl Med 1994;19(7):630635. DOI: 10.1097/00003072-199407000-00016.

2. Soundarya N, Sharada P, Prakash N, et al. Bilateral maxillary brown tumors in a patient with primary hyperparathyroidism: report of a rare entity and review of literature. J Oral Maxillofac Pathol 2011;15(1):56-59. DOI: 10.4103/0973-029X.80027.

3. Grulois V, Buysschaert I, Schoenaers J, et al. Brown tumour: presenting symptom of primary hyperparathyroidism. B-ENT 2005;1(4):191-195.

4. Phitayakorn R, Mchenry CR. Incidence and location of ectopic abnormal parathyroid glands. Am J Surg 2006;191(3):418-423. DOI: 10.1016/j.amjsurg.2005.10.049.

5. Goodman A, Politz D, Lopez J, et al. Intrathyroid parathyroid adenoma: incidence and location - the case against thyroid lobectomy. Otolarynology Head Neck Surg 2011;144(6):1-5.

6. Bahar G, Feinmesser R, Joshua BZ, et al. Hyperfunctioning intrathyroidal parathyroid gland: a potential cause of failure in parathyroidectomy. Surgery 2005;139(6):821-826.

7. Abboud B, Sleilaty G, Ayoub S, et al. Intrathyroid parathyroid adenoma in primary hyperparathyroidism: can it be predicted preoperatively? World J Surg 2007;31(4):817-823. DOI: 10.1007/s00268-006-0767-0.

8. Henry J, Sebag F, Tamagnini P, et al. Endoscopic parathyroid surgery: results of 365 consecutive procedures. World J Surg 2004;28(12):12191223. DOI: 10.1007/s00268-004-7601-3.

9. Akram K, Parker JA, Donohoe K, et al. Role of single photon emission computed tomography/computed tomography in localization of ectopic parathyroid adenoma. Clin Nucl Med 2009;34(8):500-502. DOI: 10.1097/RLU.0b013e3181abb619. 